INTERNATIONAL HIGHER EDUCATION - NUMBER 66 WINTER 2012 Pages 10-12.

Combating Unethical Behavior in Higher Education

Robin Matross Helms

Robin Matross Helms is a senior program officer at the Institute of International Education and owns Hibari Connections, an international education consulting firm based in Washington, DC. This study was sponsored by the World Bank. A longer paper on this topic is available from the author. E-mail: robin@hibariconnections.com.

Examples of unethical behavior can be found in tertiary education worldwide, in rich and poor countries alike, spanning virtually every process and function of colleges and universities-from admission to academics and research, financial management, and hiring and promotion. Such behavior hinders the effective functioning of institutions, erodes public trust, and ultimately, if left unchecked, has the potential to prevent tertiary education institution systems from fulfilling their missions and obligations to their stakeholders.

A variety of approaches are currently in use to combat unethical behavior in tertiary education. These measures fall into four categories in terms of purpose: those that aim to prevent unethical behavior, those that are designed to detect it, those that punish it once it has been detected, and those that address all three of these functions at the same time. 


\section{Preventative Measures}

Measures designed to prevent unethical behavior include standardized processes and procedures implemented by institutions and governments, as well as legislation that increases oversight of institutions or aims to prevent problematic behavior-by making it illegal. Examples include automated scoring for examinations and other standardization of admissions procedures, antidiscrimination laws and policies, and legislation that addresses fraud and other financial misconduct.

Institutions and organizations may also implement broader policies focused on morals and ethics rather than specific actions-attempting to preempt the impulse to engage in unethical behavior earlier on, by creating a culture and climate in which such behavior is not accepted. Examples include student honor codes and faculty ethics policies, set forth by institutions and disciplinary associations.

\section{MeAsures for Detecting Unethical Behavior}

Unfortunately, not all instances of unethical behavior can be prevented. In order to minimize the impact of such behavior, effective and efficient measures are needed to detect it as early as possible. In recent years, new developments in technology have come to play an important role in unveiling unethical behavior. Computer programs have been developed to detect plagiarism and verify the authenticity of degrees. Telephone "tip lines" allow individuals to call and report problematic behavior anonymously, and e-mail systems have been designed for the same purpose. Beyond detecting individual instances of problematic behavior, various organizations worldwide are using surveys to examine 
broader trends, rates, and types of unethical behaviors in a given geographic area or tertiary education system.

\section{Punitive Measures}

Measures that aim to detect corrupt and unethical behavior are only worthwhile when complemented by measures that punish such behavior once it has been discovered. The most severe of these measures is legal action, including the arrest and prosecution of offenders, as well as lawsuits that result in financial or other consequences if it is determined that the alleged perpetrator acted illegally. Such measures are applied in cases of a variety of types of unethical behavior, particularly bribery and undue influence in admissions, the production and awarding of fake degrees and other false credentials, harassment, and financial fraud and mismanagement.

When the problematic behavior does not rise to the level of legal action, career status and academic/professional sanctions may be taken by institutions. This situation is often the case in instances of academic dishonesty of certain types, which may result in failing grades and revocation of degrees for students and suspension or termination for faculty and other employees. Likewise, faculty members who engage in certain types of academic and research-related unethical behavior may be subject to professional sanctions by journals, disciplinary associations, and other academic organizations.

\section{Measures With Multiple Purposes}

Along with measures that prevent, detect, or punish unethical behavior in tertiary education, ones such as accreditation and other quality-assurance 
procedures are designed to fulfill all three of these functions. Accrediting bodies and other oversight agencies set forth operational standards and standardized procedures. When followed, such decisions serve a preventative function by reducing opportunities for individuals to engage in unethical behaviors that may corrupt the educational process and other academic and operational functions. Regular reporting requirements and periodic inspections ensure transparency and detect some aspects of problematic behavior. Sanctions imposed on institutions and individuals that are found in violation of standards and procedures fulfill the punitive function.

\section{WHAT WORKS?}

In the case of anticorruption measures, more is better. Countries that systematically fully implemented such measures have had relatively low levels of unethical behavior. The United States, for example, has a robust accreditation system, legal structures to facilitate the reasonably efficient prosecution and punishment of offenders, explicit institutional policies to impose status/career sanctions on students and employees who behave unethically, and an active reporting and press network to publicize instances of problematic behavior. Together, these measures, and the parties and stakeholders involved in implementing them, form a system of checks and balances that maximizes the chances of detecting, punishing, and ultimately preventing unethical behavior.

Of course, in countries where resources are constrained and/or where corruption is deeply entrenched, it is simply not feasible to implement all of these measures at once. Governments, systems, and institutions must prioritize measures, taking into account the overall context of tertiary education- 
historical, political, economic, etc.--in doing so. For example, in countries where corruption is centralized within the government, introducing policies that allow greater institutional autonomy and oversight of operations may help reduce unethical behavior overall. Conversely, where corruption is decentralized and institutions themselves are notoriously corrupt, increased centralization of processes, which supports an increased oversight of key functions-such as the admission process-may be more beneficial. Resources and capacity for implementation should be considered, as well. If the government does not have adequate resources to implement a high-quality admissions process, then another way to end corruption in admissions must be sought. In all cases, policies that are "on paper" only and are not feasible to implement, given available resources, should be avoided. These practices are likely to do more harm than good by demonstrating to perpetrators and potential ones that the real consequences of their behavior are minimal, thus encouraging rather than hindering unethical actions. Organizations such as the World Bank and the Organization for Economic Cooperation and Development can help governments identify "best practices" and policies that are likely to be effective in a given region.

Fortunately, even starting small can ultimately have a significant impact. Educating government and institution officials and other stakeholders about the negative consequences of unethical behavior, relatively low-cost measures, can send a clear message and begin to shift behavior. As initial measures are implemented and unethical behavior begins to decrease, even slightly, acceptance of more comprehensive measures to further decrease such behavior will likely be developed. Public trust will also increase; systems and individual 
institutions will then be perceived as more solid and will be more likely to attract private and public funding. This may constitute less competition for spaces and less corruption of the admission process and fewer instances of degree fraud. The number of faculty jobs may also increase, which may lead to less corruption in the career-management realm. Salaries are likely to be higher across the board, which may lower the incentive for bribery across all educational and administrative functions. A "virtuous cycle" is thus created; as the number of instances of corruption and other unethical behavior decreases. Confidence in systems and institutions will continue to grow; and tolerance for behavior that compromises quality and integrity will further decline. 